University of Nebraska - Lincoln

DigitalCommons@University of Nebraska - Lincoln

December 1996

\title{
Stars Classified as Constant in the General Catalogue of Variable Stars, II
}

Edward G. Schmidt

University of Nebraska-Lincoln, eschmidt1@unl.edu

Follow this and additional works at: https://digitalcommons.unl.edu/physicsschmidt

Part of the Physics Commons

Schmidt, Edward G., "Stars Classified as Constant in the General Catalogue of Variable Stars, II" (1996). Edward Schmidt Publications. 20.

https://digitalcommons.unl.edu/physicsschmidt/20

This Article is brought to you for free and open access by the Research Papers in Physics and Astronomy at DigitalCommons@University of Nebraska - Lincoln. It has been accepted for inclusion in Edward Schmidt Publications by an authorized administrator of DigitalCommons@University of Nebraska - Lincoln. 
Publications of the Astronomical Society of the Pacific 108: 1105-1111, 1996 December

\title{
Stars Classified as Constant in the General Catalogue of Variable Stars. II.
}

\author{
EDWARD G. SCHMIDT \\ Department of Physics and Astronomy, University of Nebraska, Lincoln, Nebraska 68588 \\ Electronic mail: eschmidt@unlinfo.unl.edu \\ Received 1996 July 24; accepted 1996 September 30
}

\begin{abstract}
Photometric observations have been made of 36 stars classified as CST or CST: in the General Catalogue of Variable Stars. Six of these stars are found to vary and three others were deemed possible variables. Of the certain variables, two (MR Her and TY Sge) are red pulsators, one (V1585 Cyg) is a rapid irregular variable, one is apparently a short period Cepheid-strip star (V432 Oph), one is an eclipsing binary (AQ Boo), and one is of uncertain type (V351 Cyg). The stars we have observed as MR Her and TY Sge are the same ones which were identified on finding charts in the discovery papers, suggesting that these stars have intervals of variability and intervals of quiescence. V432 Oph is of special interest because our photometry indicates that it may be either a double mode Cepheid or a peculiar long-period RR Lyrae star depending on which of a couple of possible periods turns out to be correct.
\end{abstract}

\section{INTRODUCTION}

In an earlier paper (Schmidt et al. 1992; Paper I) photometry was presented for 16 stars which were classified as constant, CST, or probably constant, CST:, in the General Catalogue of Variable Stars (the GCVS, Kholopov, 1985, 1987). The motivation for reexamining these objects was discussed there. Briefly, a few examples exist in the literature of stars which show significant changes in their pulsational properties over time scales of decades. The reason for this behavior is unknown but promises to reveal interesting stellar physics. Since stars once classified as variable but later found to be constant might fall into this category, the CST and CST: stars are a fertile ground for searching for more objects of this type.

Variables were found in the fields of three of the sixteen objects discussed in Paper I. However, in two cases, FV Del and FP Gem, the variable was not the star marked on the original finding chart. Meinunger (1993a) reexamined the Sonneburg plates which were the basis for the classifying FV Del as CST and concluded that the star we found to vary also varied on those plates; this was apparently a case of misidentification. He is currently examining the plates for FP Gem (Meinunger 1993b) to clarify its status.

The third CST star we found to vary, U Tau, turned out to be a rapid, semiregular variable. In that case, there is no possibility of misidentification and an examination of data from a century ago clearly indicated significant changes in its variations over time. Since this star is likely to be related to the T Tau stars, such behavior is not particularly surprising (see Herbst et al. 1994).

Because the earlier study did turn up several interesting stars, it was decided to continue the observation of constant stars in the Northern Hemisphere. This paper reports observations of 36 additional stars. These, together with the 16 previously observed, represent more than three-quarters of the CST and CST: stars for which observations from Behlen Observatory are feasible.

\section{THE OBSERVATIONS}

The observations were made in the interval from January 1992 (Julian Date 2,448,642) to March 1996 (Julian Date $2,450,173$ ) with the Behlen Observatory CCD photometry system. The techniques used for the observations and data analysis are identical with those described in Paper $I$ and references contained therein. A point which is relevant is that all the measurements used aperture photometry and may be subject to errors due to nearby bright stars. As before, we used the $V$ and $R$ bands and utilized differential measurements relative to other stars in the CCD field. The data were all placed on the standard system using standards from Landolt (1992) observed on photometric nights. The original observations, together with those from Paper I, will appear in the AAS CD-ROM Series, Astrophysics on Disc. The first part of the table is reproduced in Table 1 as a guide to form and content. The indices in the table are all differential relative to the comparison stars in the field. However, the mean magnitudes and colors of the comparison stars were added to place these values on the standard system.

Table 2 lists the stars observed. Column 1 gives the variable star identification while the number of nights on which the variable was observed and the number of individual observations are given in the second column. The third and fourth columns list the rms scatter of the differential $V$ and $R$ magnitudes in millimagnitudes. The magnitudes and colors on the standard system are given in the next two columns. Most of these magnitudes and colors are based on at least three photometric nights and are accurate to $0.01 \mathrm{mag}$; exceptions are indicated with colons. For the stars judged to be variable, the magnitudes and colors are a straight means of all the nights on which observations were available. The seventh column lists the range of dates of observations (given as Julian Date $-2,440,0000$ ).

Much of the decision on the variability of a particular star rests on the rms scatter in $V$ and $R$ listed in Table 2. In Fig. 1 we plot these errors against the $V$ magnitude. The rms 
TABLE 1

Photometric Data for RZ And

\begin{tabular}{lccccccr}
\hline \hline $\begin{array}{l}\text { HJD- } \\
2,440,000\end{array}$ & $V$ & $R$ & $V-R$ & $2,440,000$ & $V$ & $R$ & $V-R$ \\
\hline 8745.929 & 9.418 & 8.849 & 0.566 & 8979.563 & 9.396 & 8.801 & 0.596 \\
8755.855 & 9.437 & 8.821 & 0.614 & 8980.576 & 9.408 & 8.813 & 0.595 \\
8760.887 & 9.428 & 8.816 & 0.612 & 9612.765 & 9.432 & 8.838 & 0.593 \\
8750.872 & 9.380 & 8.833 & 0.546 & 9613.712 & 9.421 & 8.823 & 0.598 \\
8761.832 & 9.413 & 8.803 & 0.609 & & & & \\
\hline \hline
\end{tabular}

Note to TABLE 1

The full table is reproduced in the AAS CD-ROM series. The first part is shown here as a guide to form and content.

TABLE 2

Data for Stars Classified as Constant in the GCVS

\begin{tabular}{|c|c|c|c|c|c|c|c|}
\hline Star & n & $\sigma_{V}$ & $\sigma_{R}$ & $V$ & $V-R$ & Interval $\mathbf{S}$ & Status \\
\hline RZ And & 9,9 & 18 & 16 & 9.41 & $0.59:$ & $8745-9613$ & \\
\hline VZ And & 9,10 & 12 & 17 & 12.55: & $0.48:$ & $8755-9613$ & a \\
\hline X Boo & 6,6 & 11 & 15 & 10.21: & $0.49:$ & $9724-9774$ & a \\
\hline UX Boo & 15,15 & 13 & 25 & 10.43 & 0.34 & $8713-9752$ & c \\
\hline AI Boo & 15,29 & 21 & 21 & 14.56: & $0.38:$ & $8713-9774$ & a \\
\hline AQ Boo & 18,22 & 168 & 165 & 12.53 & 0.37 & $8713-9760$ & b \\
\hline RV Cnc & 7,8 & 9 & 8 & 12.78 & 0.31 & $8642-8682$ & a \\
\hline S CVn & 11,11 & 23 & 52 & 9.74 & 0.71 & $9662-9880$ & d \\
\hline $\mathrm{X} C \mathrm{CV}$ & 12,12 & 10 & 19 & 10.66 & 0.33 & $8713-8980$ & a \\
\hline SX CVn & 23,24 & 19 & 12 & 10.49 & 0.35 & $8708-9715$ & a \\
\hline WY Com & 14,14 & 20 & 34 & 15.07 & 0.38 & $8696-8980$ & a \\
\hline $\mathrm{RX} \mathrm{CrB}$ & 15,15 & 10 & 15 & 11.64 & 0.29 & $8713-9606$ & a \\
\hline YZ Cyg & 7,9 & 12 & 29 & 8.58 & -0.01 & $9907-10025$ & 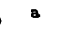 \\
\hline AK Cyg & 9,9 & 15 & 7 & 11.10 & 0.70 & $8714-8980$ & d \\
\hline CF Cyg & 9,10 & 22 & 20 & 9.18 & 0.08 & $9760-10025$ & a \\
\hline V351 Cyg & 21,38 & 66 & 39 & 14.72 & 0.93 & $8716-9936$ & b \\
\hline V578 Cyg & 10,10 & 26 & 15 & 13.67: & $0.51:$ & $9612-9687$ & $\mathbf{a}$ \\
\hline V1585 Cyg & 32,44 & 32 & 26 & 13.45 & 0.59 & $8714-9892$ & b \\
\hline ST Dra & 21,21 & 18 & 22 & 10.20 & 0.58 & $8681-9652$ & a \\
\hline UY Dra & 25,29 & 12 & 9 & 11.00 & 0.64 & 8715-9909 & d \\
\hline XZ Gem & 6,6 & 37 & 51 & 10.43 & 1.36 & $9724-9773$ & c \\
\hline V Her & 25,40 & 24 & 25 & 11.11 & 0.44 & 8715-9909 & c \\
\hline RW Her & 10,10 & 17 & 16 & 12.99: & 0.56 : & $8714-9626$ & $\mathbf{a}$ \\
\hline TZ Her & 9,9 & 17 & 27 & 8.9: & 0.26 : & $9937-10173$ & a \\
\hline MR Her & 25,35 & 300 & 199 & 12.81 & 1.79 & $8715-9936$ & b \\
\hline AC Leo & 13,30 & 93 & 49 & 9.49 & 0.54 & $9652-9774$ & d \\
\hline V432 Oph & 18,25 & 73 & 57 & 14.12 & 0.26 & $8714-9892$ & b \\
\hline RY Peg & 24,36 & 19 & 31 & 12.41 & 0.35 & 8714-9909 & d \\
\hline SZ Peg & 12,13 & 14 & 16 & 9.75 & 0.67 & $8714-9613$ & a \\
\hline UV Peg & 12,13 & 21 & 10 & 9.36 & 0.06 & $9612-9714$ & a \\
\hline TY Sge & 31,33 & 76 & 61 & 12.05 & 1.07 & 8714-9909 & b \\
\hline EL Sge & 9,9 & 11 & 25 & 10.83: & $0.25:$ & $8714-9613$ & d \\
\hline RR Ser & 13,13 & 15 & 18 & 11.37 & 0.37 : & $8713-8981$ & d \\
\hline RS Tau & 7,8 & 17 & 27 & 9.44: & 0.26 : & $9668-9714$ & a \\
\hline RZ Vir & 15,16 & 13 & 11 & 10.94 & 0.28 & $8708-8980$ & a \\
\hline VZ Vul & 12,13 & 12 & 13 & 9.56 & 0.64 & $8714-9613$ & a \\
\hline
\end{tabular}

NOTE.-The columns of this table contain the following information: Column 1, the name of the star; Column 2, the number of nights the star was observed and the total number of individual observations of the star; Columns 3 and 4 , the rms scatter of the differential observations in $V$ and $R$ expressed in milli-magnitudes; Column 5, the mean $V$ magnitude; Column 6, the mean $V-R$ index; Column 7, the range of Julian Dates on which the star was observed minus 2,440,000; Column 8, references to foot notes explaining the status of the variability of the star.

${ }^{a}$ Constant star

${ }^{b}$ Variable star. See text for discussion.

'Possible variable star. See text for discussion.

${ }^{d}$ Constant star. See text for discussion. scatter for most of the objects is less than 20 mmag in both $V$ and $R$; any which have large scatter are obvious candidates for variability. However, in assessing the scatter several factors need to be taken into account. Constant stars may show excessive scatter due to the presence of a nearby star (since we used aperture photometry). The magnitude level of a star (either too bright or too faint) and the adopted exposure time can influence the scatter. The magnitudes and number of the comparison stars can affect the errors for a particular star. A star with small scatter may still be a low-level variable.

The last column of Table 2 contains a letter which indicates the status of the variability of the stars according to the notes at the bottom of the table. When the scatter for a particular star stood out in Fig. 1, we examined the photometry to attempt to detect any small-scale variation. The factors discussed in the previous paragraph and the presence or ab-
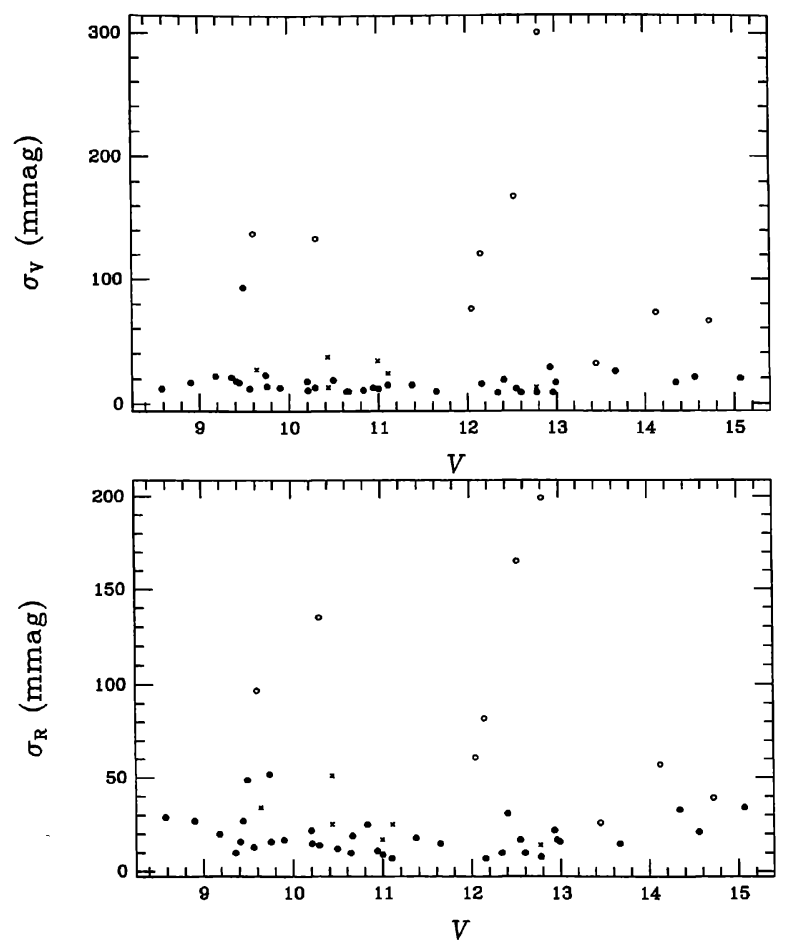

FIG. 1-The rms scatter for the stars in Table 2 and in Table 1 of Paper I plotted against the $V$ magnitudes. The filled circles represent stars which are regarded as constant, the open circles those which we concluded are variable, and the $X$ 's those which are possible variables. 

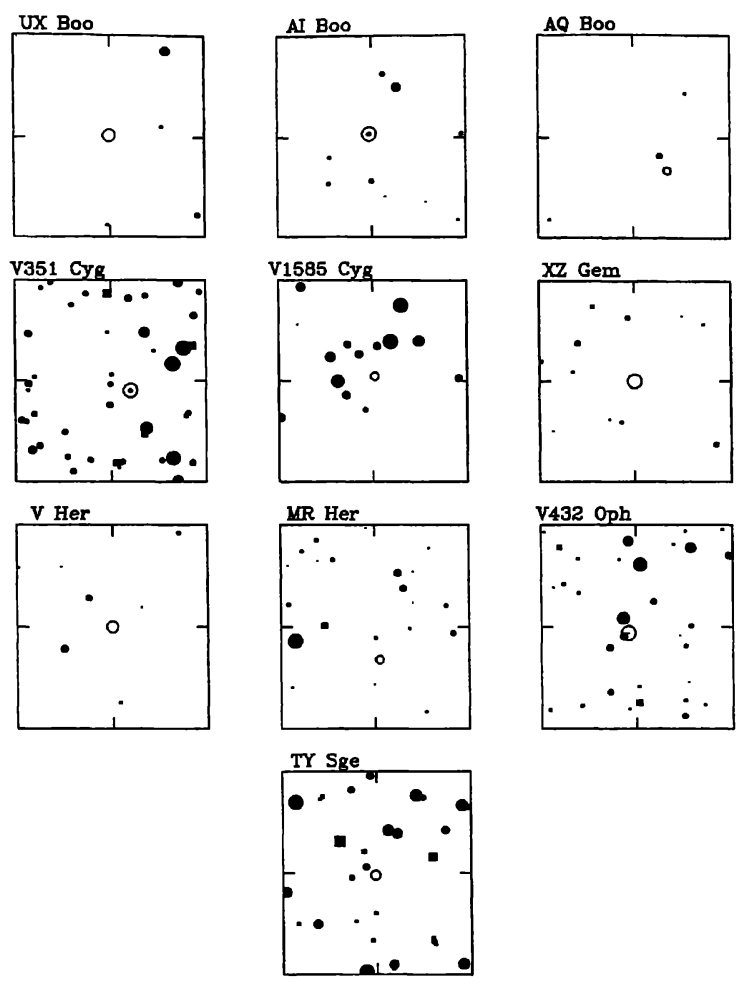

10.

12.5

15.

FIG. 2-Finding charts for the stars which were found to be variable or possibly variable. Each chart is 10 arcmin on a side. North is up and east to the left. The variables are indicated by open circles or circled points. The sizes of the circles indicate the magnitude of the stars as shown in the legend.

sence of systematic trends or periodicity in the photometry were considered in judging the variability. In a few cases we have concluded that low level variability is possible and these stars should be examined in future seasons to see if they are in the process of reestablishing variability.

Figure 2 presents finding charts for stars which are possible variables. These charts were generated from the Hubble Space Telescope Guide Star Catalogue (Lasker et al. 1990; Russell et al. 1990; Jenkner et al. 1990) as described by Schmidt and Reiswig (1993). The original finding charts were consulted for all the stars, both those in Fig. 2 and others, to verify that we are referring to the same star as was identified in the discovery paper.

\section{DISCUSSION OF INDIVIDUAL STARS}

UX Boo: The values for $\sigma_{V}$ and $\sigma_{R}$ listed in Table 2 were calculated after one point (at HJD 2448761.621) had been deleted. That point falls 0.64 mag below the others in both colors. An examination of the original data indicates no obvious problem. This leads us to suspect that this star is an eclipsing binary. Since only one of our 14 observations showed evidence of an eclipse, the eclipses must be relatively brief.

Early observers regarded this object as a W UMa star (Rugemer 1934) with a period of 2 or $3 \mathrm{hr}$ (Blazhko 1930) or

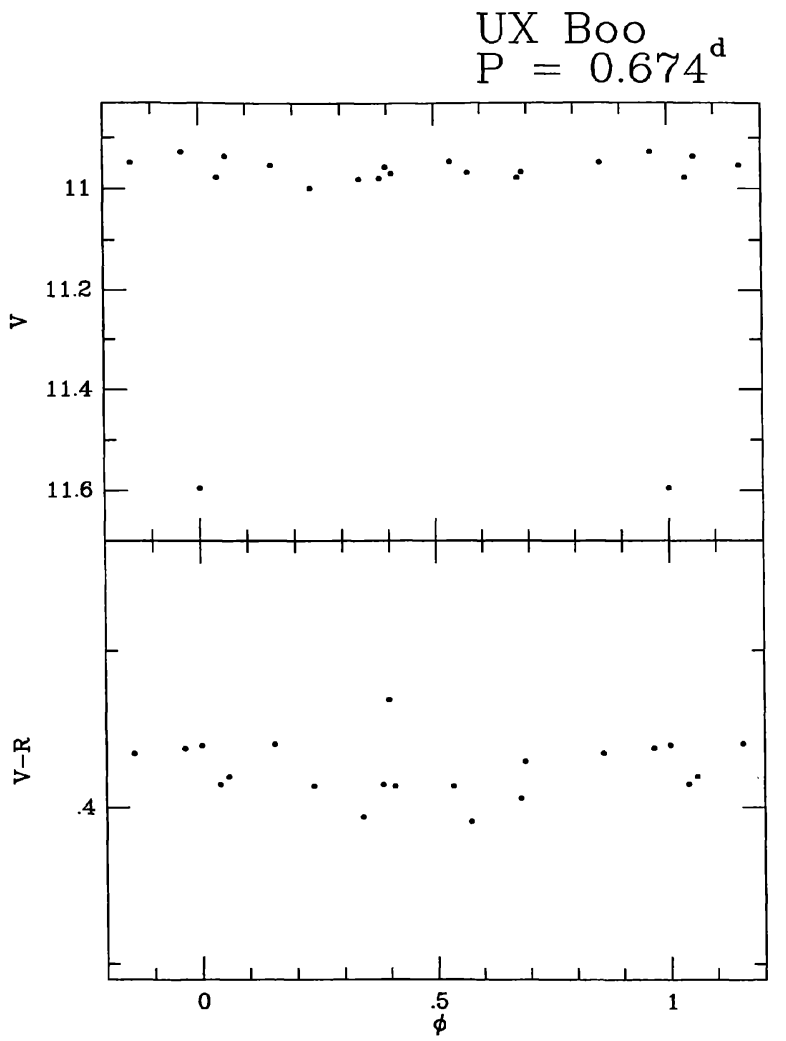

FIG. 3-Light and color curves for UX Boo plotted with a period of 0.674 and an epoch of minimum at JD 2,448,761.621.

either 0.674 or 0.504 days (Dombrovsky 1932). Our data do not rule out either of these periods and obviously we would need much more data, including a number of minima, to determine a new period. For lack of any better information, the light curves in Fig. 3 were plotted with a period of 0.674 days. Clearly, our data are inconsistent with a W UMa star.

AQ Boo: This star is variable with a total range of 0.54 in both $V$ and $R$. A period of $0.33412 \pm 0.000002$ days was derived from our data using the Discrete Data Corrected Fourier period search technique (the DDCF technique, Feraz-Mello 1981). The light curve with this period is shown in Fig. 4. It is evident that the star is an eclipsing binary. Since there is only a negligible color change, the two components must be nearly identical in temperature. Meinunger and Wenzel (1968) found this star to be constant based on 136 photographic plates. Their mean magnitude, 12.3 , is consistent with the present photometry suggesting that they observed the same star. We are unable to explain why their conclusion regarding variability does not agree with the present result.

S CVn: The rather large value of $\sigma_{R}$ is due to two low points. Since the corresponding $V$ magnitudes are not deviant, we conclude that the star is likely to be constant.

AK Cyg: This star is a visual binary. The data given in Table 2 refer to component A. For component B, our photometry gives $V=11.55, V-R=0.38$. Neither component varies in our data.

V351 Cyg: The star identified by the finding chart of 


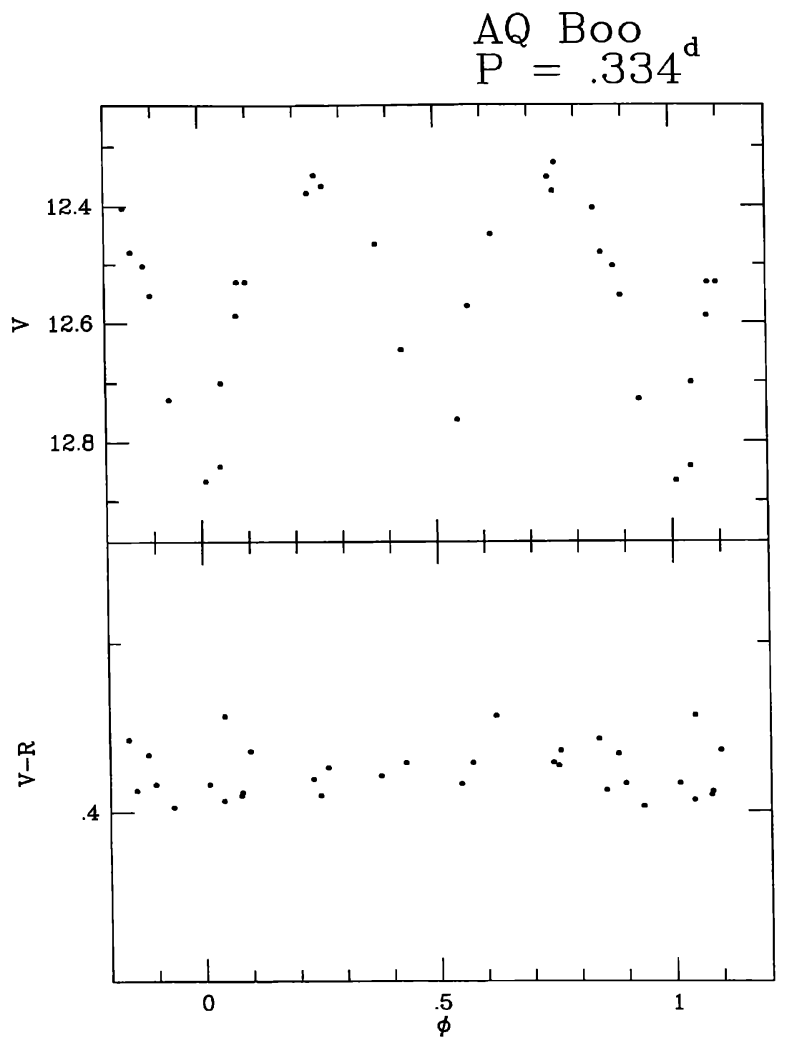

FIG. 4-Light and color curves for AQ Boo plotted with a period of 0.333412 days and epoch of minimum of HJD 2,448,716.724.

Kukarkin and Kurochkin (1942) is not variable. However, a star $65^{\prime \prime}$ west and $20^{\prime \prime}$ south of it seems to vary at the level of a couple of tenths of a magnitude. In Fig. 1, this star exhibits a rms scatter above that of other stars of similar brightness, at least in $V$. No nearby stars are visible in Fig. 2 or on the Palomar Observatory Sky Survey prints and the star is well placed on the chip. Therefore, there is no reason to suspect the data and the variation are likely to be real. We speculate that this star is V351 Cyg and that the designation of CST results from the wrong star having been marked on the original finding chart. A period search on the $V$ magnitude yielded a period of 0.104292 days. The data are plotted with that period and an epoch of minimum of $\mathrm{JD}=2,448,761.78$ in Fig. 5. It can be seen that the $V$ light curve exhibits an apparent eclipse at phase 0.00 but it is not apparent in the $R$ light curve. This peculiarity raises doubts that this is an eclipsing star and suggests that further study would be worthwhile. The scatter throughout the light curve is also a peculiarity to be settled by further observation.

V1585 Cyg: The data for this star show a range of about a tenth of a magnitude for both $V$ and $R$. A period search revealed appreciable power for a variety of periods between 0.1 days and 2.7 days. The light curve is plotted in Fig. 6 using one of these possible period, 1.1837 days. Given the scatter in the light curve and the low power, we conclude that this star is irregular in its variation with a characteristic time of the order of a day. This makes it likely that it is a

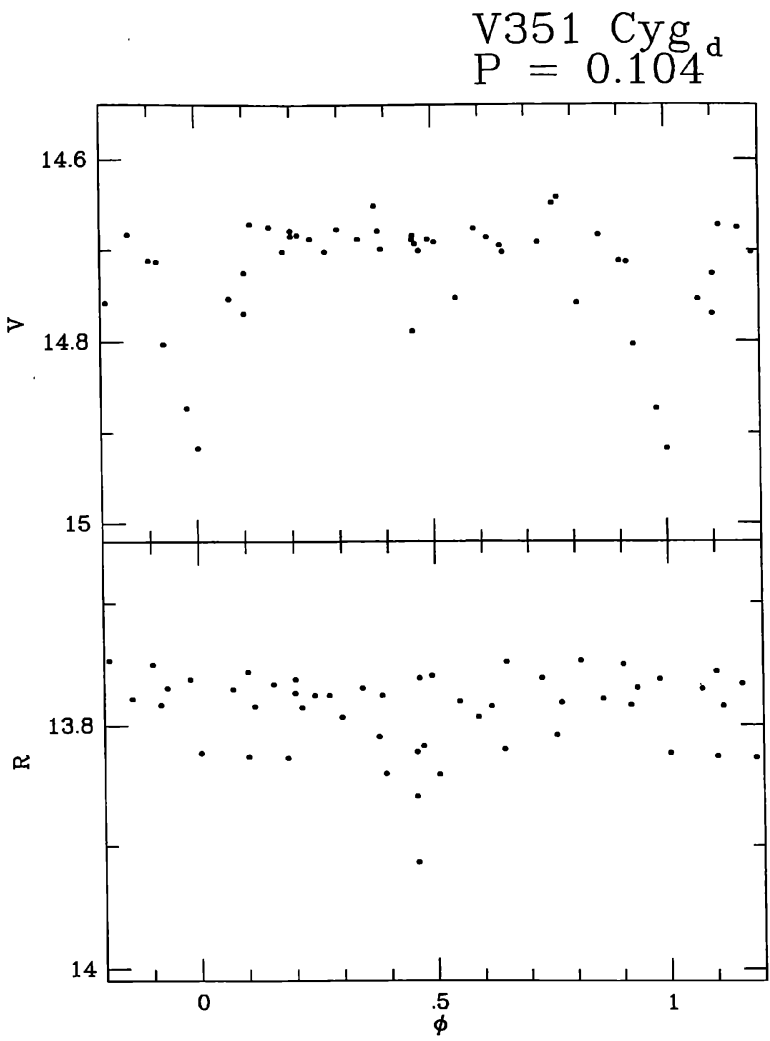

FIG. 5- $V$ and $R$ magnitudes for V351 Cyg plotted against Julian Date. A period of 0.104292 was used and zero phase was set to JD $2,448,761.78$.

rapid irregular star (type IS or IN in the GCVS) similar to $\mathrm{U}$ Tau (Paper I).

It should be noted that only the data from 1992 show good evidence for variation on this period. Those from 1994 scatter about a magnitude of $V=13.45$ except for a low point at phase 0.52 and a couple of high points near phase zero. The 1995 data are all clustered near the apparent minimum of the light curve (between phases of about 0.3 and 0.7 ). While they are consistent with the 1992 data, by themselves they do not give support to the variation. This changing behavior from one season to another is consistent with an IS or IN star.

UY Dra: This star is $18^{\prime \prime}$ on the sky from the Mira variable T Dra. We detect no variability in UY Dra and suggest that the previous report of variability which caused the star's inclusion in the GCVS was due either to scattered light from $\mathrm{T}$ Dra which may have contaminated earlier measurements or misidentification of T Dra as UY Dra.

XZ Gem: No finding chart is referenced in the GCVS but there is an isolated star at the correct coordinates and no other plausible object anywhere nearby. We conclude that this is the correct star. The dispersion in $V$ and $R$ both suggest variability. An examination of the data indicates possible variation in both bands at the level of a tenth of a magnitude. This star should be observed more closely to establish the variability.

V Her: This star was suspected of variation based on observations between HJD 2,448,715 and 2,448,761 (the 


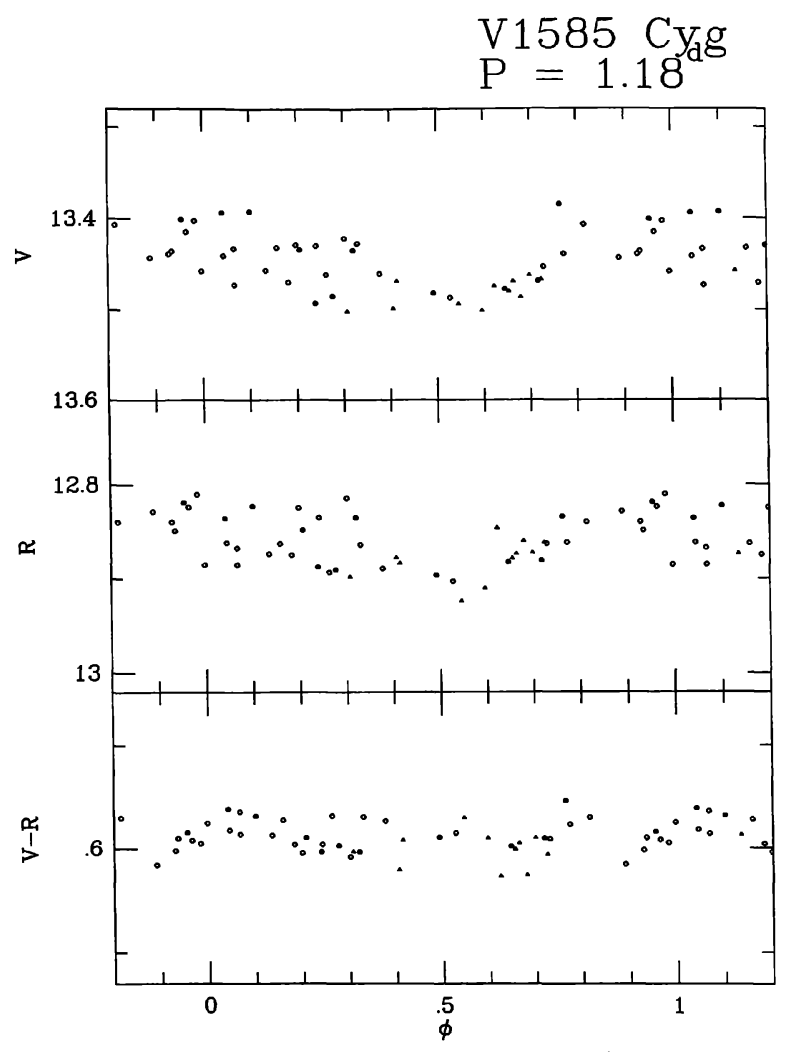

Fig. 6- Light and color curves for V1585 Cyg plotted with a period of 1.1837 days and an epoch of maximum of $\mathrm{HJD}=2,449,696.57$. The different symbols denote different observing seasons as follows: 1992, filled circles; 1994, open circles, 1995, triangles.

spring of 1992) but observations from 2,449,760 to $2,449,901$ (the spring of 1995) failed to demonstrate variability. We conclude that this star either ceased its variations after mid 1992 or a few spurious observations contaminated the 1992 data.

MR Her: This star varies on a long time scale. Our $V$ and $R$ magnitudes have ranges of 0.98 and 0.65 . Although our coverage has large gaps, it appears that a period near 200 days is likely and we plot the light curve in Fig. 7 with a period of 206 days. It can be seen that the star does not repeat from one cycle to another. This, together with the long period and very red color indicates a semi-regular variable.

Ahnert et al. (1949) describe this star's variation as consisting of rapid irregular variations for a couple of intervals of months and constant for two other intervals of about 200 days each. This behavior is consistent with a semi-regular variable and probably explains the classification in the GCVS as CST.

AC Leo: The large values of the dispersions for this star are attributable to about four observations. Since this star is near the brightness limit for our instrumentation, this may come about from instrumental effects. In any event, there is no evidence for variation above the level of a few hundredths of a magnitude.

V432 Oph: This star varies at the level of about a quarter of a magnitude in $V$. Application of the DDCF technique

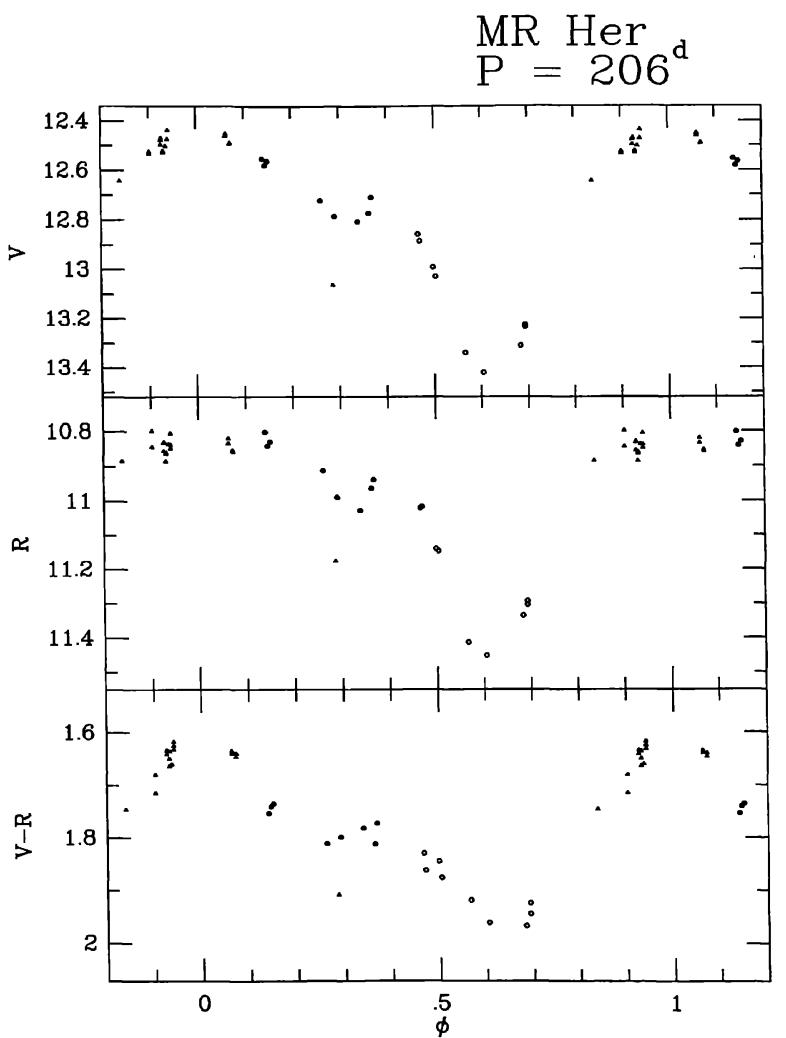

FIG. 7-Light and color curves for MR Her plotted for a period of 206 days and an epoch of maximum of $\mathrm{HJD}=2,449,922$. The various symbols indicate the dates of observation as follows: JD 2,448,715-2,448,761, filled circles; JD 2,449,605-2,449,652, open circles; JD 2,449,704-2,449,948, triangles.

yielded several periods of which 1.7315 days and 0.6325 days (which are aliases of each other) are the most likely. Examination of the light curves leads us to prefer the shorter period and we used it to plot the light curve in Fig. 8. The excess scatter around maximum light persists with any reasonable period suggesting that the star may be a double mode Cepheid if the longer period is correct or a star similar to BW Com (see Schmidt et al. 1990) if the shorter period is correct. Either possibility is interesting and this star will be observed further to resolve the ambiguity.

RY Peg: Our photometry of RY Peg shows scatter with a range of about $0.1 \mathrm{mag}$ in $V$ and $0.14 \mathrm{mag}$ in $R$. However, variations in $V$ and $R$ are uncorrelated. RY Peg is only $18^{\prime \prime}$ from the semi-regular star RZ Peg which is a magnitude brighter in $V$ and three magnitudes brighter in $R$. We conclude that the large scatter in the photometry of RY Peg is probably due to this circumstance (since we used aperture photometry) and our data provide no evidence of variability.

TY Sge: This star appears to vary with an amplitude of about a quarter of a magnitude. A period search yielded equally likely periods of 99 days and 150 days along with numerous aliases near one day. With either period there is still considerable scatter which seems to be due to nonrepeatability from one cycle to another. These factors together with its very red color lead us to conclude that it is a semi-regular variable. Its light curve is plotted in Fig. 9 with a period of 


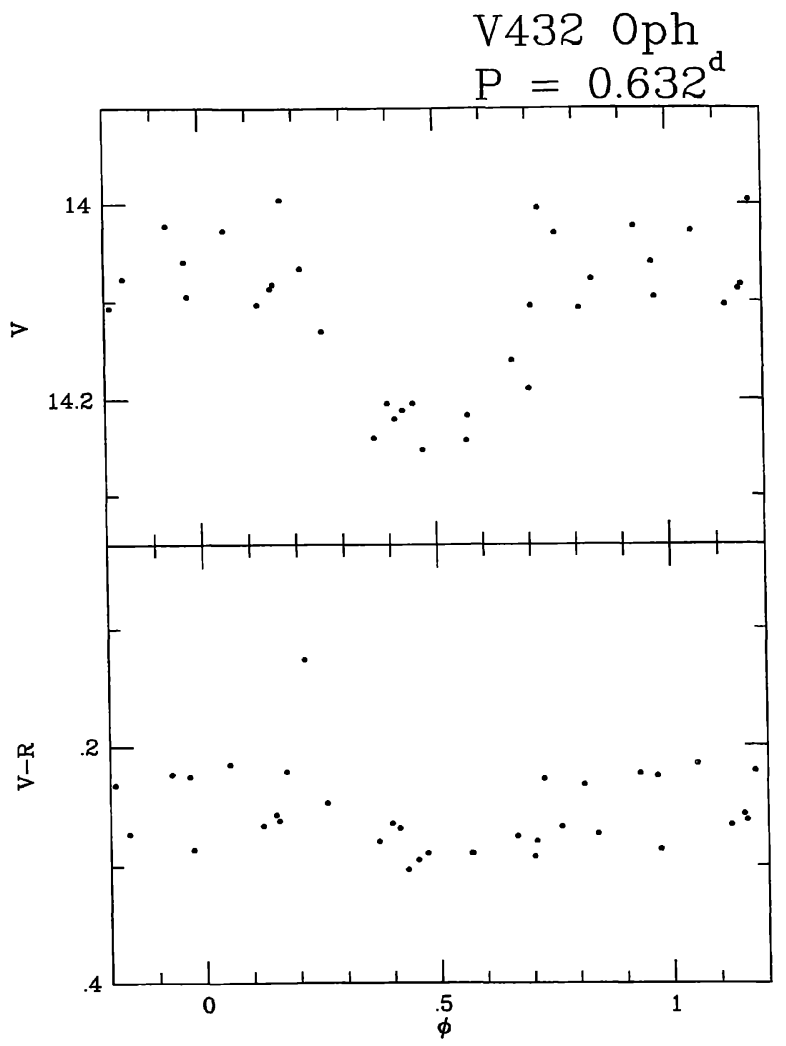

Fig. 8- Light and color curves for V432 Oph. A period of 0.6325 days and epoch of maximum of JD 2,48,755.80.

150 days. Ahnert et al. (1949) found no variations over a 500 day interval while Wolf and Wolf (1906) and Paranago (1933) reported variations of a magnitude.

EL Sge: There is some ambiguity as to the identification of this star. However, neither of the possibilities varies. The photometry refers to the star closest to the catalog coordinates.

RR Ser: Graf (1915) remarked that the identity of this star is "not entirely certain." Neither of the two possible stars varies and we give the magnitude and color for the star at the GCVS coordinates.

\section{SUMMARY AND CONCLUSIONS}

Of the 36 stars observed here, six were found to vary and an additional three were deemed possible low level variables. Considering only the certain variables, the data presented here together with that from Paper I indicate that about 17\% (nine out of 52) of stars classed as constant in the GCVS are in fact variables. Of these nine stars, four are yellow or red pulsators (FV Del, FP Gem, MR Her, and TY Sge), two are irregular variables (V1585 Cyg and U Tau), one is apparently a short period Cepheid-strip star (V432 Oph), one is an eclipsing binary (AQ Boo) and one is of unknown type (V351 Cyg).

As indicated in the introduction to Paper I, the main motivation for this project was the discovery of variable stars which have exhibited periods of quiescence in the past. In particular, we were interested in finding luminous yellow and

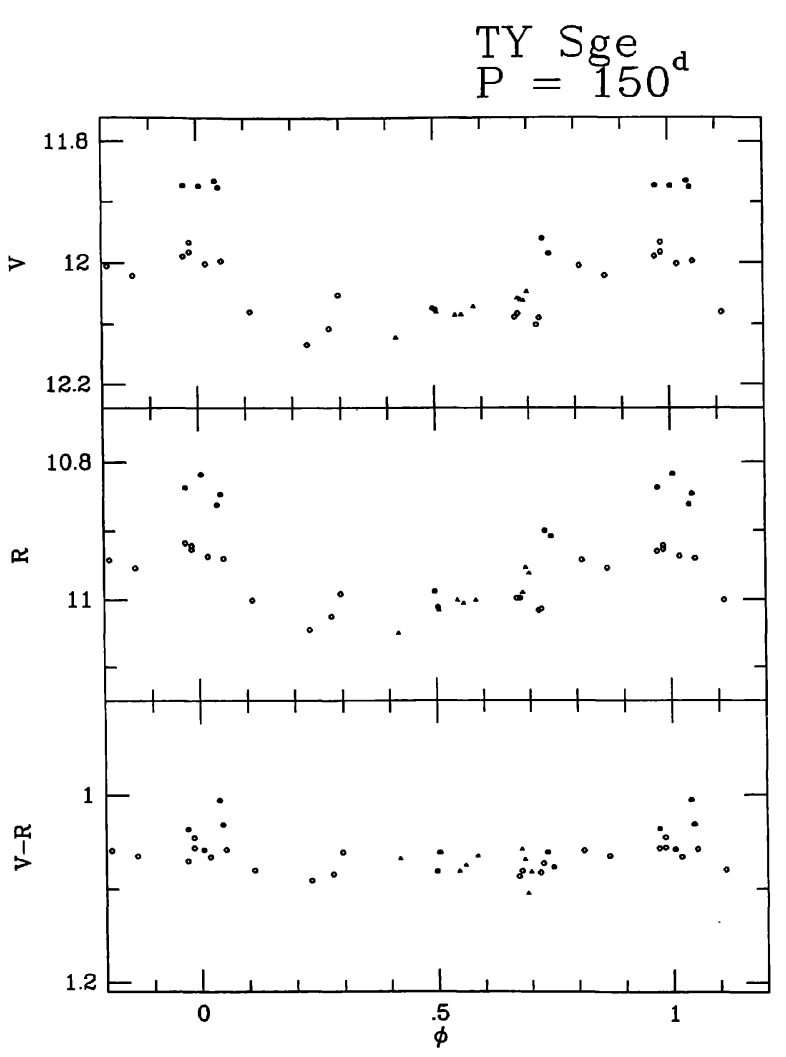

FIG. 9-Light and color curves for TY Sge plotted with a period of 150 days and an epoch of maximum of $\mathrm{HJD}=2,448,755$. The symbols denote the dates of observation as follows: JD 2,448,714-2,448,980, solid circles; JD 2,449,605-2,449,699, open circles; JD 2,449,867-2,449.909, triangles.

red pulsators, similar to R Pup and RU Cam, which show such behavior. Although we found four late type variables, the evidence was that two were the result of misidentification (FV Del, FP Gem; Paper I). However, for the two studied in the present paper, MR Her and TY Sge, there is no evidence for an error of that type. We conclude that these two stars vary in their amplitude sufficiently over a number of years to have first been discovered to vary but later were found to be constant. Both are apparently again in an interval of variability. The causes of these long term variations in pulsational behavior are unknown but may hold the clue to interesting stellar physics.

This work was supported by the National Science Foundation through Grant AST-8815806 and an REU supplement to that grant. The equipment used was funded through NSF Grant No. AST-8504072. Some of the analysis was carried out using the facilities of the Minnich Astronomical Computing Center which was established through a donation by Commander Charles B. Minnich.

\section{REFERENCES}

Ahnert, P., Hoffmeister, C., Rohlfs, E., and van de Voorde, A. 1949, Veroff. Stern. Sonneberg, 1, 161

Blazhko, S. 1930, Astron. Nach., 239, 15

Dombrovsky, S. 1932, Tashk. Circ., 19 
Feraz-Mello, S. 1981, AJ, 86, 619

Graf, K. 1915, Astron. Nach., 201, 173

Herbst, W., Herbst, D. K., Grossman, E. J., and Weinstein, D. 1994, AJ, 108, 1906

Jenkner, H., Lasker, B. M., Struch, C. R., McLean, B. J., Shara, M. M., and Russell, J. L. 1990, AJ, 99, 2082

Kholopov, P. N. 1985, General Catalogue of Variable Stars, 4th ed. (Moscow, Nauka), Vols. 1 and 2

Kholopov, P. N. 1987, General Catalogue of Variable Stars, 4th ed. (Moscow, Nauka), Vol. 3

Kukarkin, B. V., and Kurochkin, P. E. 1948, Perem. Zvezdy, 6, 74 Landolt, A. U. 1992, AJ, 104, 372

Lasker, B. M., Sturch, C. R., Mclean, B. J., Russell, J. L., Jenkner, H., and Shara, M. M. 1990, AJ, 99, 2019
Meinunger, I. 1993a, Mitt. Verf. St., 12, 163

Meinunger, I. 1993b (private communication)

Meinunger, L., and Wenzel, W. 1968, Veroff. Stern. Sonneberg, 7, 389

Paranago, P. P. 1933, Perem. Zvezdy, 7, 389

Rugemer, H. 1934, Astron. Nach., 251, 307

Russell, J. L., Lasker, B. M., Mclean, B. J., Sturch, C. R., and Jenkner, H. 1990, AJ, 99, 2059

Schmidt, E. G., Loomis, C., Groebner, A., and Potter, C. 1990, ApJ, 360, 604

Schmidt, E. G., Chab, J. R., and Reiswig, D. E. 1992, PASP, 104, 906 (Paper I)

Schmidt, E. G., and Reiswig, D. E. 1993, AJ, 106, 2429

Wolf, M., and Wolf, G. 1906, Astron. Nach., 170, 361 